\title{
A secure online image trading system for untrusted cloud environments
}

\author{
Khairul Munadi ${ }^{*}$, Fitri Arnia ${ }^{1}$, Mohd Syaryadhi ${ }^{1}$, Masaaki Fujiyoshi ${ }^{2}$ and Hitoshi Kiya ${ }^{2}$
}

\begin{abstract}
In conventional image trading systems, images are usually stored unprotected on a server, rendering them vulnerable to untrusted server providers and malicious intruders. This paper proposes a conceptual image trading framework that enables secure storage and retrieval over Internet services. The process involves three parties: an image publisher, a server provider, and an image buyer. The aim is to facilitate secure storage and retrieval of original images for commercial transactions, while preventing untrusted server providers and unauthorized users from gaining access to true contents. The framework exploits the Discrete Cosine Transform (DCT) coefficients and the moment invariants of images. Original images are visually protected in the DCT domain, and stored on a repository server. Small representation of the original images, called thumbnails, are generated and made publicly accessible for browsing. When a buyer is interested in a thumbnail, he/she sends a query to retrieve the visually protected image. The thumbnails and protected images are matched using the DC component of the DCT coefficients and the moment invariant feature. After the matching process, the server returns the corresponding protected image to the buyer. However, the image remains visually protected unless a key is granted. Our target application is the online market, where publishers sell their stock images over the Internet using public cloud servers.
\end{abstract}

Keywords: Image trading, Image matching, Secure storage, Image scrambling, DCT, JPEG

\section{Introduction}

With the advancement of the Internet, multimedia content trading has become increasingly popular. As multimedia contents, such as audio, image, and video, are available in digital form, they may be benefit from ease of manipulating, duplicating, publishing, and distributing. Despite these benefits, illegal use of multimedia data tends to grow significantly unless proper protection is implemented.

One important and challenging task in multimedia content trading, including image trading, is privacy protection (Lu et al. 2009, 2010; Premaratne and Premaratne 2012; Troncoso-Pastoriza and Perez-Gonzales 2013). Most existing work in this area has focused on access control and secure data transmission ( $\mathrm{Lu}$ et al.

\footnotetext{
*Correspondence: khairul.munadi@unsyiah.ac.id

${ }^{1}$ Department of Electrical Engineering, Syiah Kuala University, Jalan Tgk.

Syech Abdurrauf No. 7, 23111 Banda Aceh, Indonesia

Full list of author information is available at the end of the article
}

2009; Iacono and Torkian 2013). The aim is to prevent unauthorized users from accessing the data and to enable secure data exchange. However, once stored on the server, the data are left unprotected. This makes the user's private content vulnerable to untrustworthy server providers, as well as intruders.

In line with the Internet, the concept of cloud computing has also garnered increasing interest. The cloud provides computing and storage services to users via the Internet (Jeong and Park 2012). Public clouds offer these services to both organizations and individuals, but require no infrastructure or maintenance investment. Therefore, more applications and services are expected to rely on cloud resources in the future. However, privacy problems in the cloud environment need rigorous attention because the data can easily be distributed among different servers in different locations (Curran et al. 2012; Modi et al. 2013).

The Internet and cloud technology have undoubtedly pushed image trading to become commercially feasible for more individuals and small-scale business entities.

\section{Springer}

(c) 2015 Munadi et al. This article is distributed under the terms of the Creative Commons Attribution 4.0 International License (http://creativecommons.org/licenses/by/4.0/), which permits unrestricted use, distribution, and reproduction in any medium, provided you give appropriate credit to the original author(s) and the source, provide a link to the Creative Commons license, and indicate if changes were made. 
Therefore, the privacy protection of image content on the cloud server is an important consideration.

Currently, various types of images-ranging from photos, to art, graphics, and historical images-are traded online in the conventional way. The trading process has been exclusively conducted over the Internet, where images can be purchased and delivered online. Nevertheless, this conventional system has a serious drawback on the server side. Images stored on the server are left unprotected, allowing illegal access and use by untrusted server providers and intruders. Hence, a new mechanism for secure online image trading is necessary.

Based on the current practices of image trading and the wide availability of cloud servers, we argue that the following requirements should be satisfied to enable a secure image trading system running in an untrusted cloud environment:

1. The system must provide privacy protection to the stored data. Images on a cloud storage should be protected such that, even if untrusted parties break the server's access control, they cannot reach the true image content.

2. The system should provide a limited-content preview for display in various devices. To attract potential buyers, a portion of the content should be freely available for viewing. Because the display dimensions differ among devices, various reduced-size images are required.

3. The system must match the reduced-size images to the privacy-protected images.

4. The system needs to be compatible with compression standards. Because images are stored in compressed format, the image trading system should accommodate images compressed by specific standards.

Unfortunately, very few image trading schemes satisfy all these requirements. Most of the existing works (Lu et al. 2009, 2010; Premaratne and Premaratne 2012; Troncoso-Pastoriza and Perez-Gonzales 2013; Iacono and Torkian 2013; Kiya and Ito 2008; Okada et al. 2009, 2010; Liu et al. 2013; Sae-Tang et al. 2014; Zhang and Cheng 2014; Cheng et al. 2014) have separately and independently focused on a subset of these considerations.

The present paper introduces a conceptual framework for a secure image trading system in an untrusted cloud environment that satisfies all the above requirements. We focus on the Joint Photographic Experts Group (JPEG) (Wallace 1992) images, which are widely and popularly used in various applications. A trading activity involves three main parties: an image publisher, a server provider, and an image buyer. The proposed scheme facilitates secure server storage by visually protecting the publisher's images, thus preventing access to the true image content by untrustworthy server providers and unauthorized users. Reduced-size images that serve as queries are displayed on a user interface, providing a limited-content preview for potential buyers. Our target application is the online market, in which small content publishers sell their stock images over the Internet.

The remainder of the paper is organized as follows. "Related work" briefly reviews related works in the proposed research area. "Preliminaries" introduces the preliminary information, including a review on conventional repositories for image trading and their shortcomings, the Discrete Cosine Transform (DCT) and the JPEG standard, DCT-based scrambling for visual protection, and the structural similarity (SSIM) index that measures the degree of image scrambling. "Proposed framework" describes the conceptual framework of the proposed scheme. Simulation results are presented in "Simulation results". And, concluding remarks are given in "Conclusions".

\section{Related work}

The requirements formulated in "Introduction" can be divided into two main research categories: the secure storage of images on a public cloud server, and efficient image matching in visually protected (encrypted) domains for retrieval and content preview purposes.

Among the earlier works on image trading systems, the authors in Okada et al. $(2009,2010)$, Liu et al. (2013) proposed a framework that offers privacy or content protection. In their mechanism, an image is decomposed into two components with different levels of importance. One component is sent directly to a consumer; the other is first routed to an arbitrator or trusted third party (TTP) for fingerprinting and then sent to the consumer. This approach is impractical because of several reasons. First, the consumer receives two image components, increasing the memory and bandwidth usage. In addition, the approach requires a TTP and assumes that images are stored on a proprietary and trusted server.

An extension of the above proposal, which no longer separates an image into several components, was presented in Sae-Tang et al. (2014). This method specifically handles JPEG 2000 images. Although it removes image decomposition, it retains the TTP requirement, thus adding technical complexity to small content publishers.

Client-side encryptions for cloud storage have also been proposed (Iacono and Torkian 2013; Lu et al. 2009, 2010; Cheng et al. 2014). For instance, the approach in 
Iacono and Torkian (2013) encrypts the data file and changes the file structure, thus increasing the difficulties in indexing and searching of the encrypted data. In $\mathrm{Lu}$ et al. (2009, 2010) and Cheng et al. (2014), features are extracted from plaintext images and encrypted by the image owners. The encrypted features and images are then stored on a server equipped with a table of mapping relationship between them. When the user makes a query, the features from the plaintext query image are extracted and encrypted, and then sent to the server, where their similarity to the features encrypted in the database is calculated. This implies that feature extraction/encryption and image encryption are performed separately, incurring additional computational resources and complexities.

The histogram-based retrieval of Zhang and Cheng (2014) reduces the necessity of feature extraction/encryption. The images stored on a server are simply encrypted by permuting DCT coefficients and are compatible with the JPEG file format. Similarity between an encrypted query and an encrypted image is determined by calculating the distances of DCT coefficient histograms. However, this process requires nearly full JPEG decoding (up to inverse quantization) and proposes no mechanism for content preview. Therefore, how a potential buyer could select an image for purchase is not clarified.

An initial attempt to formulate a secure online image trading system was presented in Munadi et al. (2013), although no clear framework was described for a cloud environment context. This study also lacked a descriptive comparison with a conventional image trading system. Moreover, the experiments and analysis were based on a small dataset.

\section{Preliminaries}

In this section, we present some background information that is necessary to formulate our proposed framework, including a review of conventional image trading systems and their shortcomings, the DCT and JPEG standard, image scrambling in the DCT domain, and the SSIM index, which measures the degree of scrambling.

\section{Conventional model of image trading}

Most current applications that enable commercial transaction of images are strongly reliant on access control. Buyers obtain privileged access to the image repository after payment. Figure 1 illustrates a typical image repository and trading system in a conventional approach. An image publisher normally uses third-party services to host his/her commercial images. Potential buyers can browse a thumbnail collection, which provides small representations of the images. If the buyer is pleased with the image, he/she will pay an agreed price and receive an access key in return. The buyer will then be able to download the original size or full-resolution image. Alternatively, the image can be electronically sent to the buyer by the server. A practical application of this concept is best described by the digital image libraries available on several websites (KITLV; Getty Images; Corbis; iStock).

In terms of privacy, this conventional scheme is confronted with at least two serious threats or attacks that can be originated from internal and external sources, as depicted in Figure 2. The types of threats/attacks can be described as follows:

1 External threats Unauthorized users present an external threat to the image repository. Illegal access

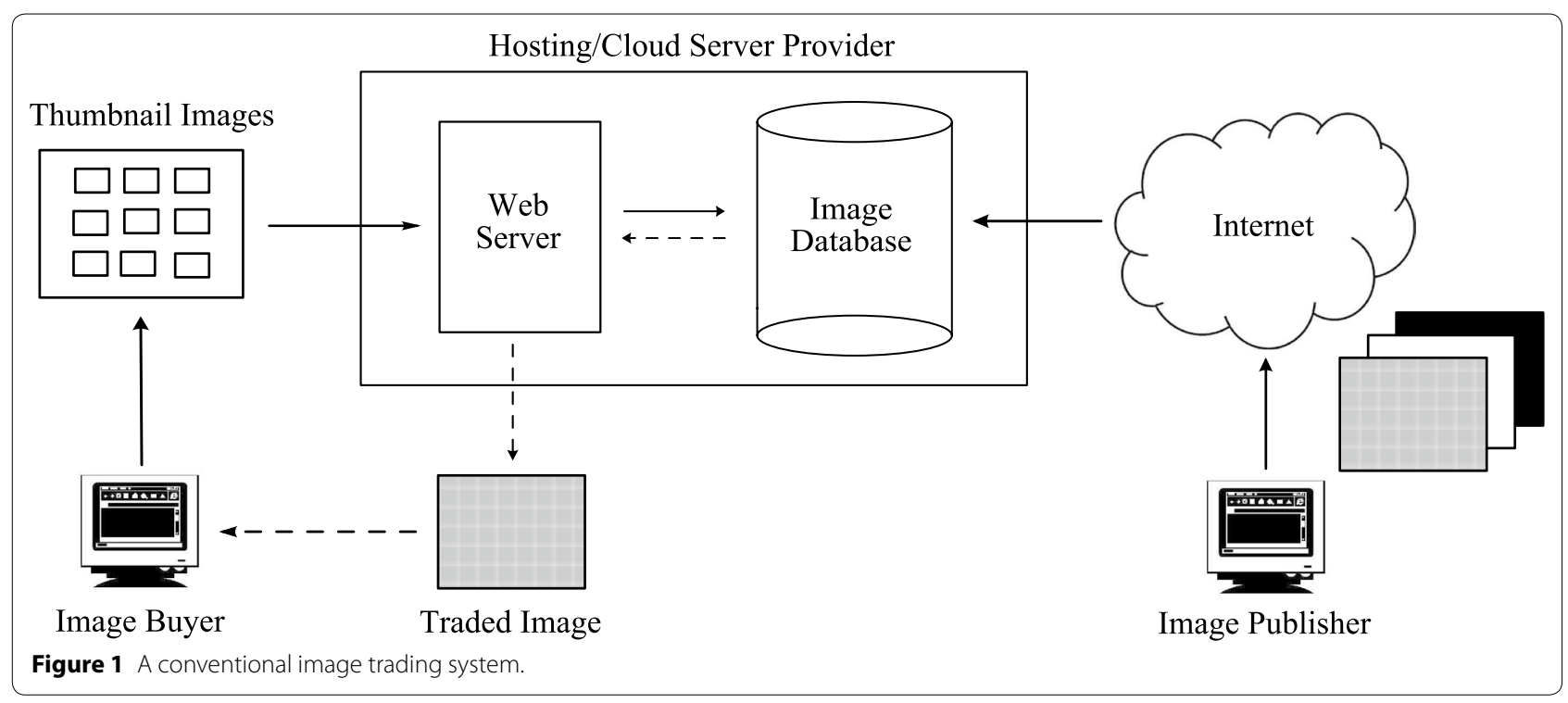




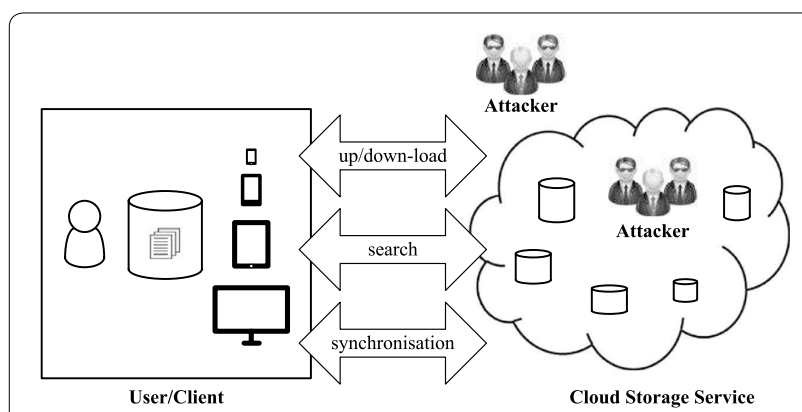

Figure 2 Source of threats/attacks in a cloud storage service, adapted from lacono and Torkian (2013).

may be obtained under various conditions, such as lack of authentication, weak access control, and malicious attacks. When access is obtained by an unauthorized user, it becomes difficult to prevent illegal use of the images.

2 Internal threats A server provider often has the highest access privileges for the stored data, such as commercial images, with no risk of detection. Therefore, a malicious provider presents an internal threat to the stored data, leading to the illegal use of images, such as theft or illegal distribution.

A cloud-based image trading framework that considers the above-mentioned issues is proposed herein. It facilitates secure storage and retrieval of original images, and prevents unauthorized parties from accessing the true content of images.

\section{DCT and JPEG}

The JPEG compression standard is based on the DCT that transforms spatial data into the frequency domain. The encoding procedure is illustrated in Figure 3 and can be summarized as follows. An original image is partitioned into $8 \times 8$ non-overlapped blocks. A function of two-dimensional Forward Discrete Cosine Transform (FDCT), as in Eq. (1), is applied to each block, resulting in $1 \mathrm{DC}$ and $63 \mathrm{AC}$ coefficients.

$$
F_{u v}=\frac{C_{u} C_{v}}{4} \sum_{i=0}^{7} \sum_{j=0}^{7} \cos \frac{(2 i+1) u \pi}{16} \cos \frac{(2 j+1) v \pi}{16} f(i, j)
$$

where

$$
C_{u}, C_{v}= \begin{cases}\frac{1}{\sqrt{2}} & \text { for } u, v=0 \\ 1 & \text { otherwise }\end{cases}
$$

For coding, an $8 \times 8$ array of the DCT coefficients is reorganized into a one-dimensional list based on a zigzag order. The order is initially started with the DC coefficient, and places the coefficients with the lowest spatial

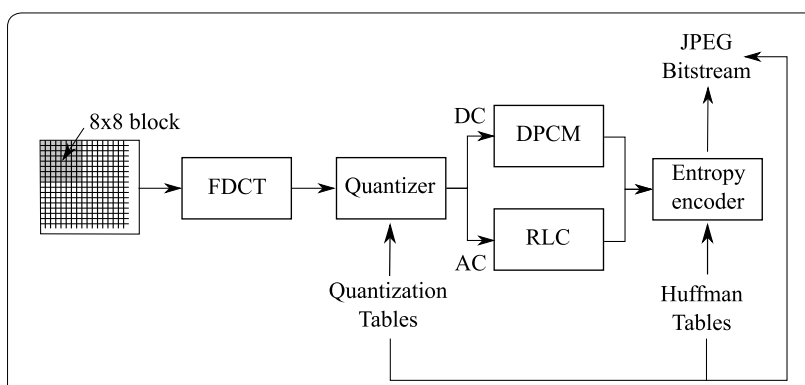

Figure 3 JPEG encoder.

frequencies in lower indices. Note that higher-frequency components generally represent the fine details of an image, and are less sensitive to human vision. Hence, they can be quantized more coarsely than the lower frequency components, and may be discarded with negligible effect on image quality. After quantization, Differential Pulse Code Modulation (DPCM) is applied to the DC coefficient, and the AC coefficients are runlength coded (RLC). As a final stage, all the coefficients are entropy encoded using Huffman or arithmetic coding. The output of the entropy encoder and some additional information, such as header and markers, form the JPEG bitstream.

\section{DCT based scrambling}

There are several approaches to visually protect the images, either in the spatial or transformed domain. Because we are dealing with the JPEG-coded images, it is preferable to consider available techniques that work in the DCT domain, such as those proposed in Weng and Preneel (2007), Khan et al. (2010a, b) and Torrubia and Mora (2003). These methods exploit the DCT coefficients to achieve various degrees of perceptual degradation, either by scrambling blocks of coefficients, or scrambling the individual DC and AC coefficients independently. The scrambling process can be further combined with an encryption technique to increase the level of protection.

The degree of perceptual degradation itself can be measured using the SSIM index. Assuming two images, $X$ and $Y$, as the comparison objects, the SSIM index is defined as follows (Wang et al. 2004; Weng and Preneel 2007):

$$
\operatorname{SSIM}(X, Y)=[l(X, Y)]^{\alpha} \cdot[c(X, Y)]^{\beta} \cdot[s(X, Y)]^{\gamma}
$$

where $X$ represents the original image and $Y$ represents the scrambled version of the original image. Functions $l(), c()$, and $s()$ correspond to luminance, contrast, and structural similarity, respectively, and $\alpha, \beta$, and $\gamma$ are the weighting factors. A simplified form of the SSIM index can be written as: 


$$
\operatorname{SSIM}(X, Y)=\frac{\left(2 \mu_{X} \mu_{Y}+C_{1}\right)\left(2 \sigma_{X Y}+C_{2}\right)}{\left(\mu_{X}^{2}+\mu_{Y}^{2}+C_{1}\right)\left(\sigma_{X}^{2}+\sigma_{Y}^{2}+C_{2}\right)}
$$

where $\mu$ is the mean intensity, $\sigma$ represents the (co)variance, and $C_{1}, C_{2}$ are numerical stability constants (Wang et al. 2004; Weng and Preneel 2007). The value of SSIM ranges from 0 to 1 , with a value of 1 indicating that $X$ and $Y$ are identical.

Samples of DCT-based scrambled images with their respective SSIM values are shown in Figure 4. As shown, different degrees of visual degradation can be obtained by applying different arrangements of the DCT coefficients. The image with the lowest SSIM value is considered the most visually protected.

\section{Proposed framework}

In this section, we describe a conceptual image trading framework for an untrusted cloud environment that satisfies all the requirements mentioned in "Introduction". The proposed framework enables secure online trading, and allows the images to be securely stored on the cloud servers after being visually protected and to be retrieved in their protected state. The following description is based on the scheme illustrated in Figure 5.

Original images owned by an image publisher are first encoded and visually protected by means of scrambling in the DCT domain (1a). At the same time, thumbnails are generated by resizing the original images to any required sizes for viewing in a display device (1b). The protected images are then uploaded and stored on a cloud repository server. In this manner, the true visual content of the original images cannot be accessed by the server provider. Thumbnails can be stored on the same server, and are publicly accessible through the website. A potential image buyer will browse the thumbnail library and choose images of interest, which also serve as queries (2). When a query image is submitted, the thumbnail is matched with the protected images by comparing the moment invariants of the thumbnail and of the DC-image generated from the protected images (3). After this matching

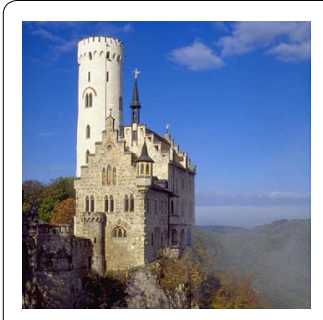

Original image

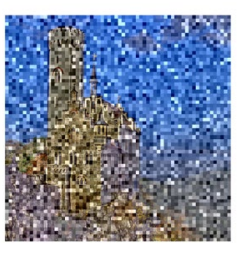

$\mathbf{a}$

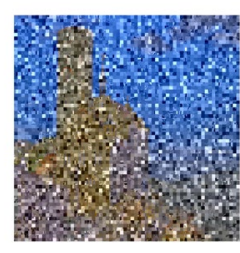

b

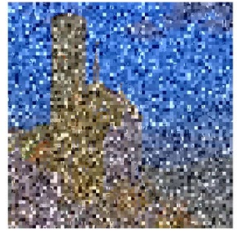

c

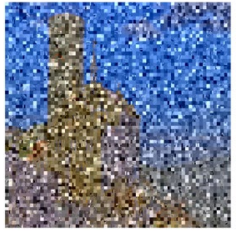

d

Figure 4 Sample of scrambled images. a DC coefficients are scrambled, SSIM $=0.3882$. b Blocks of AC coefficient are scrambled, SSIM $=0.1464$. c Blocks of $8 \times 8$ coefficients are scrambled, SSIM $=0.1519 . \mathbf{d}$ DC and AC coefficients are separately scrambled, SSIM $=0.1435$.

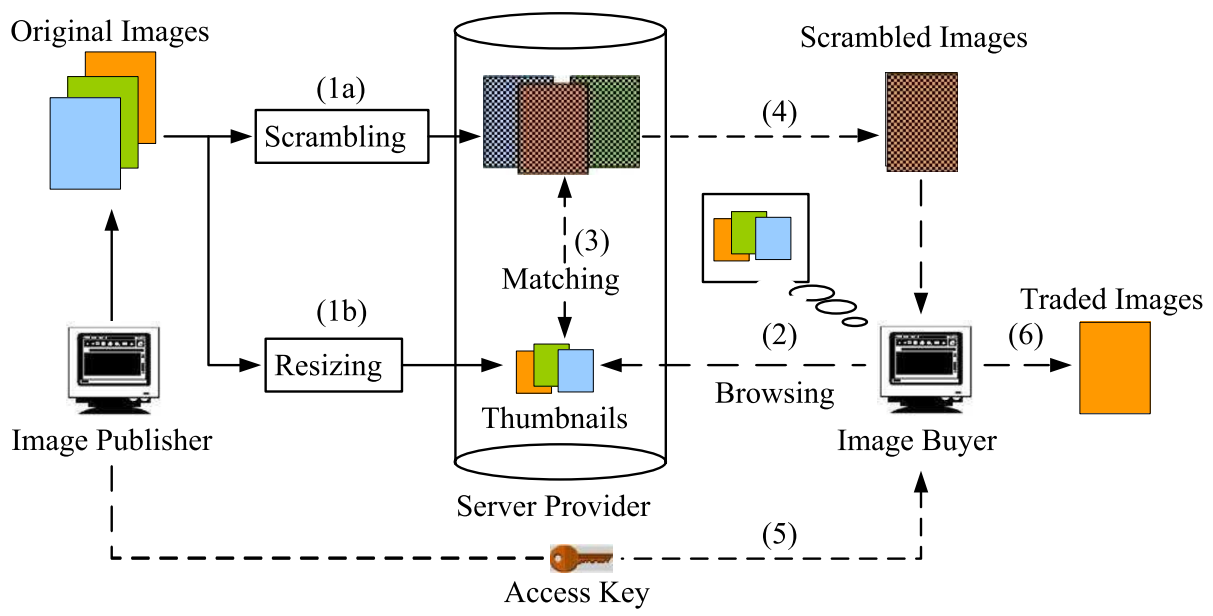

Figure 5 Proposed framework. 
process, the server will return the matched image, which can then be downloaded or sent to the potential buyer (4). However, the matched image remains visually protected unless a key is granted by the image publisher after payment or other authorization (5). Using an authentic key, the buyer will be able to decode and descramble the data, resulting in the true traded image (6).

\section{Scrambling process}

The main purpose of image scrambling is to provide visual protection so that the true content is perceptually meaningless or degraded. Therefore, the images are secure against ill-intentioned parties who may have access to the server, such as a hosting provider or hackers. Depending on the degree of scrambling, visual protection can be achieved by applying existing scrambling techniques that work in the DCT domain, such as those proposed in Kiya and Ito (2008) and Khan et al. (2010a, b).

A simplified diagram of a JPEG-based image scrambling for visual protection is shown in Figure 6, in which a block-based permutation is applied to the quantized DCT coefficients. Descrambling is simply a reverse process, given the same key as in the scrambling proses is available.

\section{DC image generation and thumbnails}

It is known that the DC coefficient of each $8 \times 8$ array of DCT coefficients is actually an average value of the 64 pixels within the corresponding block. Hence, it contains very rich visual information. An image constructed from DC components is a reduced-sized version that is visually similar to the original. Therefore, the DC image itself is a rich feature descriptor that can be exploited for matching purposes.

The process of generating a DC-image from DCT coefficients is illustrated in Figure 7. Initially, an image is partitioned into $8 \times 8$ non-overlapped blocks (referred to as a tile or a block), and a forward DCT function is employed to each block. The DC coefficient of each block represents the local average intensity and holds most of the block energy. DC coefficients from all of the blocks are then arranged according to the order of the original blocks, resulting in a reduced-size image $\left(\frac{1}{64}\right.$ of the original image) referred to as a DC-image.

In relation to the JPEG standard, it is worth noting that the DC coefficients can be directly extracted from the

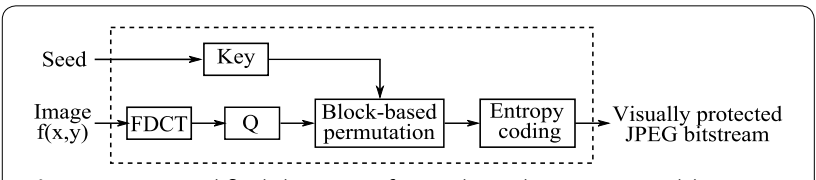

Figure 6 A simplified diagram of JPEG-based image scrambling.

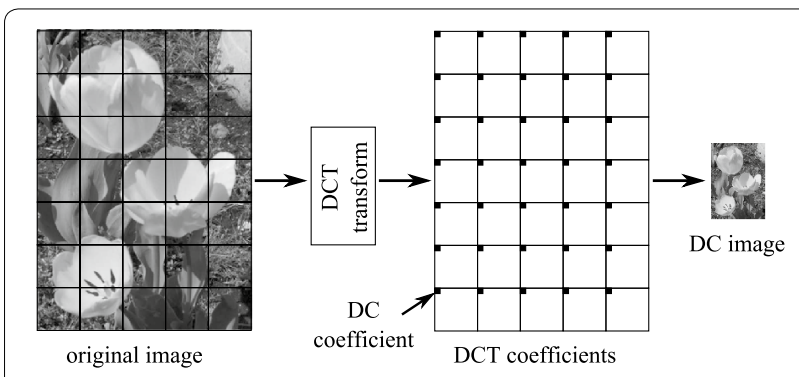

Figure 7 DC-image generation.

JPEG bitstream without the need for full JPEG decoding (Arnia et al. 2009), and the DC-image can be generated accordingly.

However, thumbnails for preview or browsing purposes can be produced by downscaling the original images to the sizes best suited to the dimensions of the display devices.

\section{Image matching}

In this section, an image matching technique and its corresponding matching distance are described. We exploit the seven $\mathrm{Hu}$ moments (Ming-Kuei 1962) for matching purposes. The moments of an image, with pixel intensities $I(x, y)$ and of size $M \times N$, are defined by:

$$
m_{p q}=\sum_{y=0}^{M-1} \sum_{x=0}^{N-1} x^{p} y^{q} I(x, y)
$$

Rather than Eq. (4), the central moments:

$$
\mu_{p q}=\sum_{y=0}^{M-1} \sum_{x=0}^{N-1}(x-\bar{x})^{p}(y-\bar{y})^{q} I(x, y)
$$

with

$$
\bar{x}=\frac{m_{10}}{m_{00}}, \quad \bar{y}=\frac{m_{01}}{m_{00}}
$$

are often used, which are invariant to translation. Furthermore, normalized central moments are defined by:

$$
\eta_{p q}=\frac{\mu_{p q}}{\mu_{00}^{\gamma}}
$$

with

$$
\gamma=\frac{p+q}{2}+1, \quad p+q=2,3, \ldots
$$

and these are also invariant to changes in scale. Algebraic combinations of these moments can provide more attractive features. The most popular are those offered by $\mathrm{Hu}$, which are independent of various transformations. Hu's 
original moment invariants (Ming-Kuei 1962; Huang and Leng 2010) are given by:

$$
\begin{aligned}
M_{1}= & \mu_{20}+\mu_{02} \\
M_{2}= & \left(\mu_{20}-\mu_{02}\right)^{2}+4 \mu_{11}^{2} \\
M_{3}= & \left(\mu_{30}-3 \mu_{12}\right)^{2}+3\left(\mu_{21}+\mu_{03}\right)^{2} \\
M_{4}= & \left(\mu_{30}+\mu_{12}\right)^{2}+\left(\mu_{21}+\mu_{03}\right)^{2} \\
M_{5}= & \left(\mu_{30}-3 \mu_{12}\right)\left(\mu_{30}+\mu_{12}\right)\left[\left(\mu_{30}\right.\right. \\
& \left.\left.+\mu_{12}\right)^{2}-3\left(\mu_{21}+\mu_{03}\right)^{2}\right] \\
& +3\left(\mu_{21}-\mu_{03}\left[3\left(\mu_{30}+\mu_{12}\right)^{2}-\left(\mu_{21}+\mu_{03}\right)^{2}\right]\right. \\
M_{6}= & \left(\mu_{20}-\mu_{02}\right)\left[\left(\mu_{30}+\mu_{12}\right)^{2}\right. \\
& \left.-\left(\mu_{21}+\mu_{03}\right)^{2}\right]+4 \mu_{11}\left(\mu_{30}\right. \\
& \left.+\mu_{12}\right)\left(\mu_{21}+\mu_{03}\right) \\
M_{7}= & \left(3 \mu_{21}-\mu_{03}\right)\left(\mu_{30}+\mu_{12}\right) \\
& \times\left[\left(\mu_{30}+\mu_{12}\right)^{2}-3\left(\mu_{21}+\mu_{03}\right)^{2}\right] \\
& +\left(\mu_{30}-3 \mu_{12}\right)\left(\mu_{21}+\mu_{03}\right)\left[3\left(\mu_{30}+\mu_{12}\right)^{2}\right. \\
& \left.-\left(\mu_{21}+\mu_{03}\right)^{2}\right]
\end{aligned}
$$

Image matching, between thumbnails and visually protected images, involves calculating the moment distance, $d$, between the thumbnails and the DC component of the visually protected images. We define the distance as:

$$
d(a, b)=\sum_{j=1}^{7}\left|M_{j}^{a}-M_{j}^{b}\right|
$$

where, $a$ and $b$ denote the thumbnail and the DC image, respectively, and $M$ represents Hu's moments. The matching process proceeds as follows:

1. The moments of a thumbnail image are calculated.

2. DC coefficients from each block of the visually protected JPEG bitstream are extracted to generate the DC image.

3. The moments of the DC images are calculated.

4. The moment distances between the query and the DC images are calculated using Eq. (7). The minimum value of $d(a, b)$ corresponds to image matching.

\section{Key sharing}

Once authorization has been requested, a corresponding scramble key is sent to the buyer by the image publisher. The true image content is accessible to the image buyer after proper decoding that includes the unscrambling process using the given key. Various options are available for delivering the scramble key to a buyer. For instance, it could be attached to the system and use the same cloud server or a system built in a different and independent server, or could be accomplished by other online means, such as email.

\section{Simulation results}

Simulations were mainly conducted to verify the matching performance between thumbnails of various sizes that serve as query images and their corresponding DCimages extracted from the visually protected images. These images were assumed to be stored on the server and available for trading. The moment distance defined in Eq. (7) was used as the matching metric.

\section{Simulation conditions}

The experiment was conducted using a dataset of 100 images with an original size of $512 \times 512$ pixels. Ten samples used as query images are shown in Figure 8. Using four different thumbnail sizes for viewing, four separate experiments were carried out. In each experiment, thumbnails were generated by rescaling the original images by a factor of $0.125,0.1875,0.25$, and 0.391 . This resulted in images of size $64 \times 64,96 \times 96,128 \times 128$, and $200 \times 200$ pixels, respectively.

As described in "DCT based scrambling", block-based scrambling of the DCT coefficients was performed to produce visually protected images. For simplicity, we scrambled only blocks of AC coefficients while preserving the original position of the DC coefficients. The size of the DC images, constructed using the DC coefficients of the protected images, was $64 \times 64$ pixels. These protected images and thumbnails were assumed to be stored on the same server.

Figure 9 shows an example of the images generated in the simulations. The image size was scaled to represent thumbnails for content preview (browsing), a DC image, and a visually protected image. For comparison purposes, we also calculated the distance between the thumbnails and the visually protected images.

\section{Results}

The results of each set of query images are presented in Tables 1, 2, 3 and 4. There are 100 matching runs presented in each table. The first two tables present the matching distances between the thumbnails (query images) and the visually protected images, and the last two present the matching distances between the thumbnails (query images) and the DC images generated from the visually protected images. Simulations using a dataset of 100 images with four different sizes of query images resulted in 40,000 matching attempts between the thumbnails and the visually protected images, and 40,000 matching attempts between the thumbnails and the DC images. 


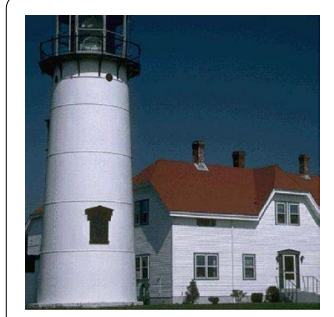

Image 1

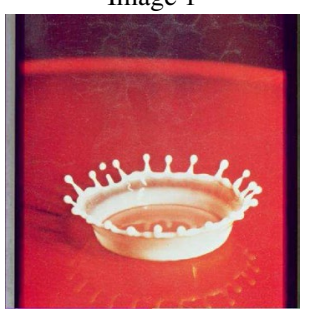

Image 6

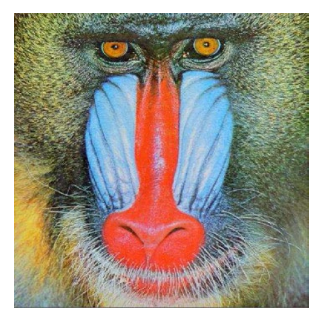

Image 2

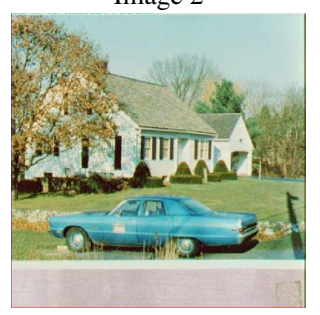

Image 7

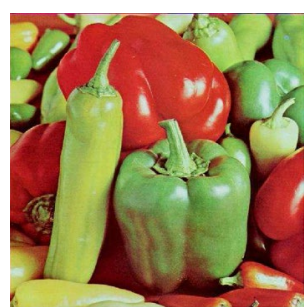

Image 3

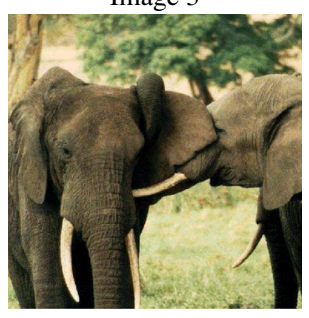

Image 8

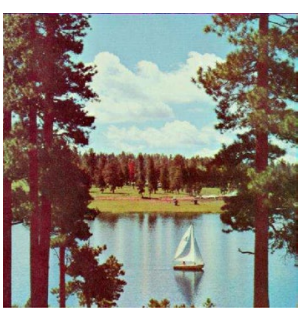

Image 4

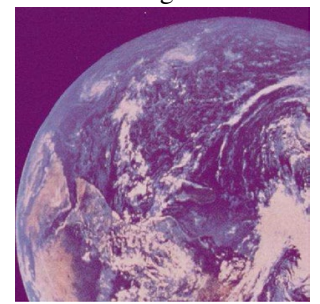

Image 9

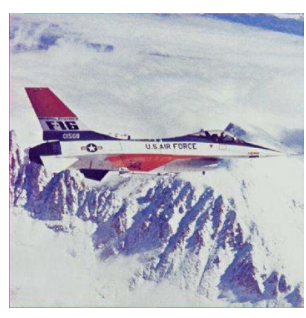

Image 5

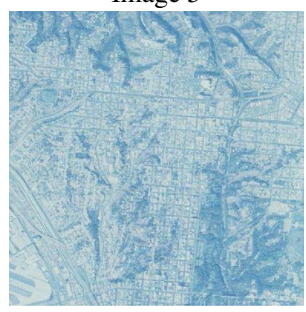

Image 10

Figure 8 Ten sample images taken from a dataset of 100 images and used as queries in the simulation.

In Tables 1 and 2, we present the matching distances between the thumbnails and visually protected images. The sizes of the thumbnails are $64 \times 64$ and $200 \times 200$ pixels, respectively. As can be seen, the distance values vary and are much higher than zero. These results confirmed that the visual content of the thumbnails and of their corresponding visually protected images is no longer identical after DCT-based scrambling. Moreover, the proposed distance measure is not applicable to a direct matching between a thumbnail and a visually protected image.

Table 3 summarizes the matching results between the thumbnail and the DC images of the same size. In this case, the displayed image for browsing and the DC image generated from the visually protected image were the same size, i.e., $64 \times 64$ pixels. In contrast to the above results, the distances between the thumbnails and their corresponding DC images were very close to zero (bold values), i.e., less than 0.2 .

The matching results between the thumbnail and DC images of different sizes are presented in Table 4. In this case, the thumbnail took its largest size, $200 \times 200$ pixels, whereas the size of the DC image was $64 \times 64$ pixels. Similar to the results in Table 3, the distance values were very small (bold values). Note that the distance values between all thumbnails of various sizes and the DC images were close to zero. This is confirmed by the averaged value of all the matching distances, as presented in Table 5.

From the above results, we can make several concluding observations. Despite its simplicity, the proposed system offers both visual protection and a content preview

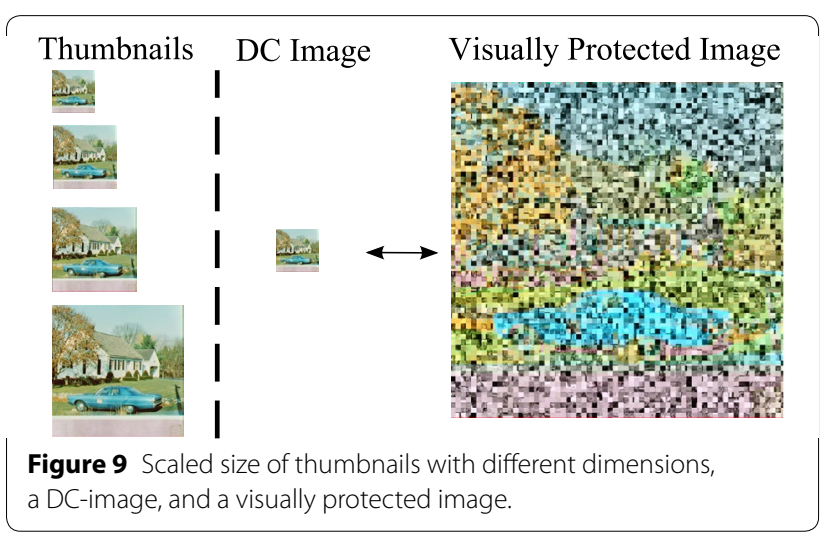

of the traded images. The proposed moment distance performed satisfactorily in retrieving the target images, with all queries for each experiment returning the correct visually protected images. This means that the matching performance was not affected by the variation in thumbnail size. Thus, thumbnails could be adjusted according to the size of display device.

\section{Conclusions}

We have presented a conceptual framework for secure online image trading in a cloud environment. The traded images were visually protected in the DCT domain, and stored on an untrusted server. Thumbnails of original images were publicly accessible through the website and served as queries. Image matching between the thumbnails and protected images was achieved by comparing the moment invariants of the thumbnails and of the 

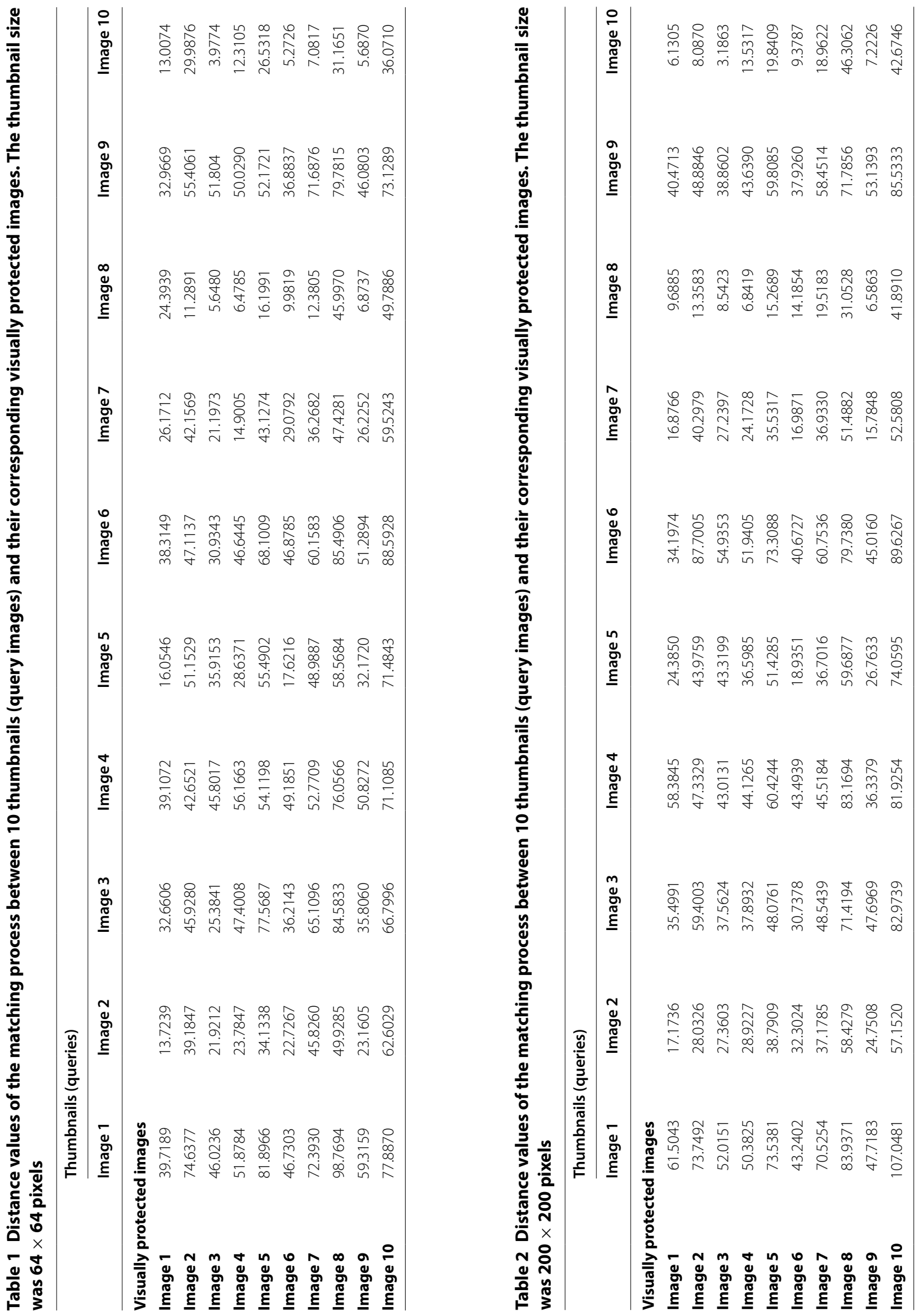

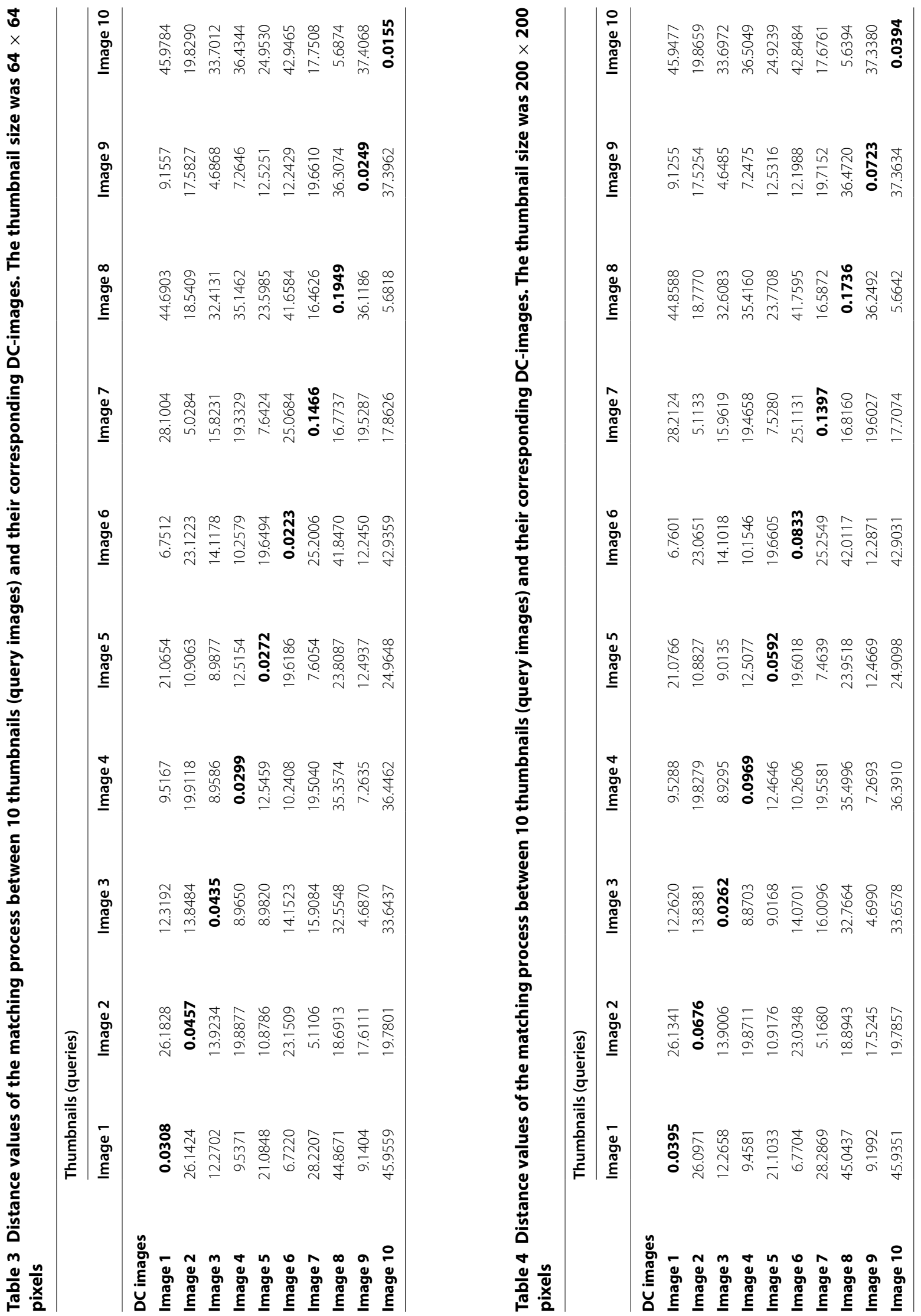
Table 5 Averaged distance values between all the thumbnails (query images) of various sizes and their corresponding DC images

\begin{tabular}{llll}
\hline Thumbnail size & & & \\
\hline $64 \times 64$ & $96 \times 96$ & $128 \times 128$ & $200 \times 200$ \\
\hline 0.0548 & 0.0449 & 0.0648 & 0.0635 \\
\hline
\end{tabular}

Each size represents an average of 100 values.

DC-image generated from the protected images. The proposed moment distance enabled the target images to be differentiated from other protected images in the database.

\section{Authors' contributions}

KM conceived the conceptual framework. KM, MF, and HK developed the research design. FA and MS prepared and ran the simulations, KM and FA wrote the paper. KM, MF, and HK reviewed the paper. All authors read and approved the final manuscript.

\section{Author details}

1 Department of Electrical Engineering, Syiah Kuala University, Jalan Tgk. Syech Abdurrauf No. 7, 23111 Banda Aceh, Indonesia. ${ }^{2}$ Graduate School of System Design, Tokyo Metropolitan University, 6-6 Asahigaoka, Hino-shi, Tokyo 191-0065, Japan

\section{Acknowledgements}

The work reported in this paper is the result of research projects partially funded by the Directorate General of Higher Education (DGHE) of the Republic of Indonesia, under the International Research Collaboration and Scientific Publication Scheme year 2014

\section{Compliance with ethical guidelines}

\section{Competing interests}

The authors declare that they have no competing interests.

\section{Image source disclosure}

Most of images used in this paper are obtained from the USC-SIPI Image Database (http://sipi.usc.edu/database/), which is freely available for research purposes. The database is maintained by Signal and Image Processing Institute, the University of Southern California. Copyright information of the images can be found at http://sipi.usc.edu/database/copyright.php.

Received: 16 September 2014 Accepted: 20 May 2015

Published online: 17 June 2015

\section{References}

Arnia F, Munadi K, Fujiyoshi M, Kiya H (2009) Efficient content-based copy detection using signs of DCT coefficient. In: IEEE symposium on industrial electronics and applications, 2009 (ISIEA 2009), vol 1, pp 494-499, 4-6 Oct 2009

Cheng B, Zhuo L, Bai Y, Peng Y, Zhang J (2014) Secure Index Construction for Privacy-Preserving Large-Scale Image Retrieval. In: Proceedings of IEEE fourth international conference on big data and cloud computing (BdCloud), pp 116-120

Corbis. http://www.corbisimages.com/. Accessed 1 Sept 2014

Getty Images. http://www.gettyimages.com/. Accessed 1 Sept 2014
Huang Z, Leng J (2010) Analysis of Hu's moment invariants on image scaling and rotation. In: Proceedings of IEEE ICCET, Chengdu, China, pp 476-480

lacono LL, Torkian D (2013) A system-oriented approach to full-text search on encrypted cloud storage. In: International conference on cloud and service computing (CSC), pp 24-29

iStock. http://www.istockphoto.com/. Accessed 1 Sept 2014

Jeong H, Park J (2012) An efficient cloud storage model for cloud computing environment. In: Proceedings of international conference on advances in grid and pervasive computing, vol 7296, pp 370-376

Curran K, Carlin S, Adams M (2012) Security issues in cloud computing. In: Cloud computing for teaching and learning: strategies for design and implementation. IGl Global, Hershey, Pennsylvania, USA, pp 200-208

Kiya H, Ito I (2008) Image matching between scrambled images for secure data management. In: Proceedings of 16th EUSIPCO, Lausanne, Switzerland, August 25-29, 2008

KITLV, Universiteit Leiden. Digital Image Library. http://media-kitlv.nl/. Accessed 1 Sept 2014

Khan MI, Jeoti V, Khan MA (2010a) Perceptual encryption of JPEG compressed images using DCT coefficients and splitting of DC coefficients into bitplanes. In: 2010 international conference on intelligent and advanced systems (ICIAS), ICIAS2010, Kuala Lumpur, Malaysia, pp 1-6, 15-17 June 2010

Khan MI, Jeoti V, Malik AS (2010b) On perceptual encryption: variants of DCT block scrambling scheme for JPEG compressed images. In: Kim T-H, Pal SK, Grosky WI, Pissinou N, Shih TK, Slezak D (eds) FGIT-SIP/MulGraB, communications in computer and information science. vol 123, Springer, New York, pp 212-223

Liu SC, Fujiyoshi M, Kiya H (2013) An image trading system using amplitudeonly images for privacy- and copyright-protection. IEICE Trans Fundam E96-A(6):1245-1252

Lu W, Varna AL, Swaminatahan A, Wu M (2009) Secure image retrieval through feature protection. In: Proceedings of IEEE ICASSP, pp 1533-1536

Lu W, Varna AL, Swaminatahan A, Wu M (2010) Security analysis for privacy preserving search of multimedia. In: Proceeding of IEEE ICIP, Hongkong, pp 2093-2096

Ming-Kuei H (1962) Visual pattern recognition by moment invariants. IRE Trans Inf Theory 8:179-187

Modi C, Patel D, Borisaniya B, Patel A, Rajarajan M (2013) A survey on security issues and solutions at different layers of cloud computing. J Supercomput 63:561-592

Munadi K, Syaryadhi M, Arnia F, Fujiyoshi M, Kiya H (2013) Secure online image trading scheme using DCT coefficients and moment invariants feature. In: Proceedings of IEEE 17th international symposium on consumer electronics (ISCE), Taiwan, pp 291-292

Okada M, Okabe Y, Uehara T (2009) Security analysis on privacy-secure image trading framework using blind watermarking. In: Proceedings of IEEE ninth annual international symposium on applications and the internet, pp 243-246

Okada M, Okabe Y, Uehara T (2010) A web-based privacy-secure content trading system for small content providers using semi-blind digital watermarking. In: Proceedings of annual IEEE consumer communications and networking conference, Las Vegas, USA, pp 1-2

Premaratne P, Premaratne $M$ (2012) Key-based scrambling for secure image communication. In: Gupta P, Huang D, Premaratne P, Zhang X (eds) Emerging intelligent computing technology and applications. Springer, Berlin, pp 259-263

Sae-Tang W, Liu S, Fujiyoshi M, Kiya H (2014) A copyright- and privacyprotected image trading system using fingerprinting in discrete wavelet domain with JPEG 2000. IEICE Trans Fundam E97-A(1):2107-2113

Troncoso-Pastoriza JR, Perez-Gonzales F (2013) Secure signal processing in the cloud: enabling technologies for privacy-preserving multimedia cloud processing. IEEE Signal Process Mag 30(2):29-41

Torrubia A, Mora F (2003) Perceptual cryptography of JPEG compressed images on the JFIF bit-stream domain. In: Proceedings of international conference on consumer electronics (ICCE), pp 58-59

Wallace GK (1992) The JPEG still picture compression standard. IEEE Trans Consum Electron 38(1):xviii-xxxiv 
Wang Z, Bovik AC, Sheikh HR, Simoncelli EP (2004) Image quality assessment: from error visibility to structural similarity. IEEE Trans Image Process 13(4):600-612

Weng L, Preneel B (2007) On encryption and authentication of the DC DCT coefficient. In: Proceedings of the second international conference on signal processing and multimedia applications (SIGMAP), pp 375-379
Zhang X, Cheng H (2014) Histogram-based retrieval for encrypted JPEG images. In: Proceedings of IEEE China Summit and international conference on signal and information processing (ChinaSIP), pp 446-449

\section{Submit your manuscript to a SpringerOpen ${ }^{\odot}$} journal and benefit from:

- Convenient online submission

- Rigorous peer review

- Immediate publication on acceptance

- Open access: articles freely available online

- High visibility within the field

- Retaining the copyright to your article

Submit your next manuscript at $\$$ springeropen.com 